Discrete Comput Geom 26:549-571 (2001)

DOI: $10.1007 / \mathrm{s} 00454-001-0022-2$

\title{
Recognition of Flat Orbifolds and the Classification of Tilings in $\mathbb{R}^{3}$
}

\author{
O. Delgado-Friedrichs \\ Fakultät für Mathematik, Universität Bielefeld, \\ Postfach 100131, 33501 Bielefeld, Germany \\ delgado@mathematik.uni-bielefeld.de
}

\begin{abstract}
We discuss criteria for an orbifold to carry a flat geometry, i.e., one which is modelled on euclidean geometry, and show how these lead to a practical flatness test in the three-dimensional case. This has immediate applications in combinatorial tiling theory, namely in the classification of three-dimensional periodic tilings up to equivariant equivalence, and in the material sciences.
\end{abstract}

\section{Introduction}

According to Thurston [Th2], a $d$-dimensional orbifold is a space which is (compatibly) locally modelled after $\mathbb{R}^{d}$ modulo finite group actions. Its singular locus is the set of points on which the group action is non-trivial. The underlying space is stratified according to the essentially different actions which occur. A cellular subdivision of the underlying space is admissible with respect to the orbifold structure if each open cell is contained in some stratum. As with manifolds, it is often useful to describe an orbifold in terms of the combinatorial structure of an admissible cellular subdivision, e.g., a triangulation, together with appropriate information on the groups associated to each cell.

The question then arises whether a given orbifold is good, i.e., is covered-in the sense of an orbifold covering - by some simply connected manifold and, if so, whether there is a compatible geometry on that manifold.

In combinatorial tiling theory, we represent periodic tilings by special triangulations of the orbifolds derived as orbit spaces of their respective symmetry groups. These special orbifold triangulations, or, more precisely, the particular combinatorial descriptions which turned out to be useful, are called Delaney symbols and were introduced by Dress in the mid-1980s [Dr1], [Dr2]. Based on Delaney symbols, systematic methods for the classification, generation and visualization of tilings in two and three dimensions have been developed and implemented [DH1], [De1], [Hu2], [BH], [DH3]. These have been successfully applied in the solutions of numerous classification tasks, as, e.g., [DH2], 
[DHZ], [Hu3], [DHM], [DH5]. The 1-skeleton of a periodic three-dimensional tiling can be interpreted as the atom-bond-network of a potential crystal. We have found that many known crystal networks - and of course many new and possibly interesting structurescan be obtained in this way from relatively simple tilings [ $\left.\mathrm{DDH}^{+}\right]$, [DH5]. Thus practical enumeration methods for three-dimensional tilings have direct implications in the material sciences.

The basic idea of the Delaney symbol approach seems to be a very natural one, as it has been reinvented several times. Most notably, there is the theory of coloured graphs, crystallizations and GEMs (graph-encoded manifolds) as established by Pezzana and developed further by Ferri, Gagliardi, Lins and others (see [LM] and [Li]). Delaney symbols can be regarded as a generalization of GEMs to the orbifold case.

A central problem in combinatorial tiling theory is to determine whether a given wellformed symbol gives rise to a tiling in the desired geometry. In two dimensions this can be solved by simple criteria based on the Euler characteristic and the well-known classification of good two-dimensional orbifolds (see [Vi]). The three-dimensional problem is much harder, even if we restrict our attention to tilings of euclidean space. In effect, we have to decide whether a given 3-orbifold represented by a Delaney symbol is covered by $\mathbb{R}^{3}$ in such a way that all homeomorphisms of $\mathbb{R}^{3}$ which are annihilated by the covering projection are euclidean isometries.

The main theoretical result of this paper is that, indeed, this can be done, so, at least in principle, tilings of three-dimensional space can be generated effectively (see Theorem 15). In a second part, we consider strategies to obtain an algorithm which is fast enough to be actually useful. More details on this, including complexity results, will be given in a forthcoming paper.

Many commonly used representations of orbifolds can be easily converted to admissible triangulations, and these in turn can be converted to Delaney symbols. Thus, our approach to the flatness problem is of relevance not only to tiling theory.

In the following section we introduce definitions and notation. We present and discuss a general scheme for flatness tests in Section 3 and its application to the three-dimensional case in Sections 4 and 5. In Sections 6-8 we discuss a practical approach for dimension 3. Finally, in Sections 9, we illustrate our methods by an example.

\section{Delaney Symbols}

Delaney symbols have been discussed and applied in many papers. Here we give a short introduction and state some basic facts for reference.

Consider a simply connected $d$-manifold $X$ (without boundary) together with a regular CW-filtration $\mathcal{X}=\left\{X_{0}, \ldots \mathcal{X}_{d}\right\}, \emptyset=X_{-1} \subset X_{0} \subset X_{1} \subset \cdots \subset X_{d}=X$. Such a CWfiltration is called a tiling of $X$. Let $\Gamma$ be a group acting on $X$ by self-homeomorphisms and respecting the filtration, i.e., satisfying $\gamma\left(X_{i}\right)=X_{i}$ for all $\gamma \in \Gamma$ and all $i=0, \ldots, d$. There are obvious induced actions of $\Gamma$ on the sets of cells of any given dimension and on the set of inclusion chains of cells. In particular, there is an action of $\Gamma$ on the set of flags (maximal chains)

$$
\mathcal{F}:=\left\{F=\left(F_{0}, \ldots, F_{d}\right) \in \prod_{i=0}^{d} \pi_{0}\left(X_{i}-X_{i-1}\right) \mid F_{i-1} \subseteq \overline{F_{i}} \text { for } i=1, \ldots, d\right\} .
$$


We can, on the other hand, use the fact that for each flag $F=\left\{F_{0}, \ldots, F_{d}\right\}$ and each $i \in\{0, \ldots, d\}$ there is a unique flag $F^{\prime}=\left\{F_{0}^{\prime}, \ldots, F_{d}^{\prime}\right\}$ with $F_{j}=F_{j}^{\prime}$ if and only $j \neq i$, to define a second group action on $\mathcal{F}$. Let

$$
\Sigma=\Sigma_{I}:=\left\langle\sigma_{0}, \ldots, \sigma_{d} \mid \sigma_{0}^{2}=\cdots=\sigma_{d}^{2}=1\right\rangle
$$

denote the free Coxeter group with index set $I:=\{0, \ldots, d\}$. As a shortcut in notation, we usually skip the braces when giving $I$ explicitly, thus $\Sigma_{0,1,2}:=\Sigma_{\{0,1,2\}}$.

A right action of $\Sigma$ on $\mathcal{F}$ is obtained by defining for all $F \in \mathcal{F}$ and all $i \in I$ the image $F \sigma_{i}$ as the unique flag which differs from $F$ in exactly its $i$-dimensional component. The actions of $\Gamma$ and $\Sigma$ on $\mathcal{F}$ commute, so the $\Sigma$-action carries over to the orbit space $\mathcal{D}=\mathcal{D}_{\mathcal{X}, \Gamma}:=\Gamma \backslash \mathcal{F}:=\{\Gamma F \mid F \in \mathcal{F}\}$.

In the case where $\Gamma$ acts freely on $X$, the $\mathrm{CW}$-complex $\mathcal{X}$ is determined uniquely up to compatible homeomorphisms by the abstract $\Sigma$-set $\mathcal{D}$. If $\Gamma$ does not act freely, additional information specifying the stabilizers of each cell is needed. It turns out to be sufficient to provide a map

$$
\begin{aligned}
M=M_{\mathcal{X}, \Gamma}: \mathcal{D} \times I \times I & \rightarrow \mathbb{N}, \\
(D, i, j) & \mapsto m_{i, j}(D),
\end{aligned}
$$

where $m_{i, j}(D):=r_{i, j}(C):=\inf \left\{r>0 \mid C\left(\sigma_{i} \sigma_{j}\right)^{r}=C\right\}$ for some $C \in D$ (note that the value of $m_{i, j}(D)$ does not depend on the choice of $C$ ). The quotient $v_{i, j}(D):=$ $m_{i, j}(D) / r_{i, j}(D)$ is sometimes called a branching limit or branching number. It is easy to see that a branching limit $v>1$ indicates a rotational symmetry of order $v$.

The pair $\left(\mathcal{D}_{\mathcal{X}, \Gamma}, M_{\mathcal{X}, \Gamma}\right)$ is called the Delaney symbol of the $\Gamma$-equivariant CWfiltration $\mathcal{X}$ on $X$.

From now on, we are mostly concerned with generalized formal Delaney symbols$D$-symbols for short-by which we mean pairs $(\mathcal{D}, M)$ where $\mathcal{D}$ is a transitive $\Sigma_{I}$-set for some finite index set $I \subset \mathbb{N}$ and $M$ is a map from $\mathcal{D} \times I \times I$ into $\mathbb{N}$ such that the following obvious conditions hold for all $D \in \mathcal{D}$ and all $i, j \in I$ :

(DS0) $m_{i, i}(D)=1$.

(DS1) $m_{i, j}(D)=m_{i, j}\left(D \sigma_{i}\right)=m_{j, i}(D)$.

(DS2) $D\left(\sigma_{i} \sigma_{j}\right)^{m_{i, j}(D)}=D$.

We regard D-symbols as a generalized discrete analogue of orbifolds. A morphism (or, to stress the analogy, a covering) between D-symbols $(\mathcal{D}, M)$ and $\left(\mathcal{D}^{\prime}, M^{\prime}\right)$ is a map $\varphi: \mathcal{D} \rightarrow \mathcal{D}^{\prime}$ satisfying $\varphi\left(D \sigma_{i}\right)=(\varphi(D)) \sigma_{i}$ and $m_{i, j}(\varphi(D))=m_{i, j}(D)$ for all $D \in \mathcal{D}$ and all $i, j \in I$. A functor TOP (for topological realization) from the category of Dsymbols back into the category of topological spaces is obtained by associating to each element a copy of the (\#I - 1)-dimensional standard simplex with facets labelled by the elements of $I$ and identifying the $i$-labelled facets of two simplices whenever the corresponding D-symbol elements are interchanged by $\sigma_{i}$. The identification is to be done in such a way that the remaining labels match, so $\operatorname{TOP}(\mathcal{D})$ is uniquely determined up to homeomorphisms. Accordingly, the dimension of a D-symbol is defined as one less than the cardinality of its index set $I$. The topological realization of a D-symbol morphism is defined as the direct product with the identity map on the standard simplex modulo identifications. It is easy to see that the result is a well-defined continuous map. 
The analogue to universal covers is provided by what we call the universal preimage. A D-symbol $(\widetilde{\mathcal{D}}, \widetilde{M})$ is called a universal preimage of a $\mathrm{D}$-symbol $(\mathcal{D}, M)$ with respect to base points $\widetilde{D} \in \widetilde{\mathcal{D}}$ and $D \in \mathcal{D}$ if there is a morphism $\varphi:(\widetilde{\mathcal{D}}, \widetilde{M}) \rightarrow(\mathcal{D}, M)$ which maps $\widetilde{D}$ to $D$ such that for any morphism $\psi:\left(\mathcal{D}^{\prime}, M^{\prime}\right) \rightarrow(\mathcal{D}, M)$ and any fixed $D^{\prime} \in \psi^{-1}(D)$ there is a uniqe $\varphi^{\prime}:(\widetilde{\mathcal{D}}, \widetilde{M}) \rightarrow\left(\mathcal{D}^{\prime}, M^{\prime}\right)$ with $\varphi^{\prime}(\widetilde{D})=D^{\prime}$ and $\varphi=\psi \circ \varphi^{\prime}$.

By the usual arguments, a universal preimage must —up to isomorphism-be unique and independent of the chosen base points. Its existence is established by the following construction: for some fixed $D \in \mathcal{D}$, consider the subgroup

$$
\Lambda=\Lambda_{D}(\mathcal{D}, M):=\left\langle\tau\left(\sigma_{i} \sigma_{j}\right)^{m_{i, j}(D \tau)} \tau^{-1} \mid \tau \in \Sigma ; i, j \in I\right\rangle \leq \Sigma .
$$

Now, let $\widetilde{\mathcal{D}}:=\Lambda \backslash \Sigma:=\{\Lambda \tau \mid \tau \in \Sigma\}$, the set of cosets of $\Lambda$ in $\Sigma$, which is a transitive $\Sigma$-set via the usual action $(\Lambda \tau) \rho=\Lambda(\tau \rho)$, and set

$$
\begin{aligned}
\varphi: \Lambda \backslash \Sigma & \rightarrow \mathcal{D}, \\
\Lambda \tau & \mapsto D \tau .
\end{aligned}
$$

Obviously, $\Lambda$ is contained in the $\Sigma$-stabilizer of $D$, so by standard arguments, $\varphi$ is a welldefined $\Sigma$-map. We define $\widetilde{M}$ by $m_{i, j}(\Lambda \tau):=m_{i, j}(D \tau)$, so $\varphi$ fulfils the requirement for a morphism of D-symbols. To show that $(\widetilde{\mathcal{D}}, \widetilde{M})$ is indeed a D-symbol, we note that (DS0) and (DS1) are immediate, while for each $\Lambda \tau \in \widetilde{\mathcal{D}}$ and each pair $i, j \in I$, the group element $\tau\left(\sigma_{i} \sigma_{j}\right)^{m_{i, j}(D \tau)} \tau^{-1}$ is, by definition, in the stabilizer of $\Lambda \in \widetilde{\mathcal{D}}$, so $\left(\sigma_{i} \sigma_{j}\right)^{m_{i, j}(D \tau)}=\left(\sigma_{i} \sigma_{j}\right)^{m_{i, j}(\Lambda \tau)}$ is in the stabilizer of $\Lambda \tau$, which proves (DS2). Universality follows from the fact that $\Lambda$ is indeed defined such as to be contained in the $\Sigma$-stabilizer of any preimage of $D$ by a D-symbol morphism.

As with orbifolds, the universal cover, or, in this case, the topological realization of the universal preimage, may or may not be a manifold. For orbifolds, even if the underlying space is a manifold, there may be non-trivial local group actions. In a D-symbol we have similar phenomena, which we subsume under the following definitions:

Definition 1. A D-symbol $(\mathcal{D}, M)$ is called unwound if, for each $D \in \mathcal{D}$ and all $i, j \in I$, we have $v_{i, j}(D)=1$ or, equivalently, $D\left(\sigma_{i} \sigma_{j}\right)^{r} \neq D$ for $0<r<m_{i, j}(D)$. It is called loopless if $D \sigma_{i}$ is always different from $D$. A D-symbol is called semi-good if its universal cover is unwound.

The universal cover is always loopless. In fact, it is easy to see that it is always oriented, meaning that there is a function $d: \widetilde{\mathcal{D}} \rightarrow\{-1,+1\}$ with $d\left(D \sigma_{i}\right)$ never equal to $d(D)$.

Definition 2. A D-symbol $(\mathcal{D}, M)$ with universal preimage $(\widetilde{\mathcal{D}}, \widetilde{M})$ is called good if it is semi-good and $\operatorname{TOP}(\widetilde{\mathcal{D}}, \widetilde{M})$ is a manifold.

The smallest example of a non-semi-good $\mathrm{D}$-symbol is given as follows: let $\mathcal{D}:=$ $\{A, B\}$ and $I:=\{0,1,2\}, A \sigma_{0}=A \sigma_{1}=A, B \sigma_{0}=B \sigma_{1}=B, A \sigma_{2}=B, m_{0,2}(A)=$ $m_{1,2}(A)=2, m_{0,1}(A)=1$ and $m_{0,1}(B)=k>1$. The universal preimage of $(\mathcal{D}, M)$ (which has four elements) corresponds to the so-called teardrop orbifold, a 2-sphere with a single singular "cone" point. 
The fundamental group of a D-symbol $(\mathcal{D}, M)$ is defined as the group $\pi_{1}(\mathcal{D}, M)$ of all automorphisms $\alpha$ of the universal preimage satisfying $\varphi \circ \alpha=\varphi$, where $\varphi:(\widetilde{\mathcal{D}}, \widetilde{M}) \rightarrow$ $(\mathcal{D}, M)$ is the canonical projection, as above. It can be shown that $\pi_{1}(\mathcal{D}, M)$ is isomorphic to $\Sigma_{D} / \Lambda_{D}(\mathcal{D}, M)$, where $\Sigma_{D}$ is the $\Sigma$-stabilizer of $D \in \mathcal{D}$. Balke and Valverde have described a finite presentation of this group for finite $\mathcal{D}$ in [BV], which is a good starting point for practical calculations.

We are now in a position to state the flatness problem in the context of D-symbols:

Definition 3. Ad-dimensional D-symbol $(\mathcal{D}, M)$ with universal preimage $(\widetilde{\mathcal{D}}, \widetilde{M})$ and fundamental group $\pi_{1}(\mathcal{D}, M)$ is called flat or euclidean if it is semi-good and there is a homeomorphism

$$
\alpha: \operatorname{TOP}(\widetilde{\mathcal{D}}, \tilde{M}) \rightarrow \mathbb{R}^{d}
$$

such that for all $\rho \in \pi_{1}(\mathcal{D}, M)$ the map $\alpha \circ \operatorname{TOP}(\rho) \circ \alpha^{-1}$ is an isometry with respect to the usual euclidean metric on $\mathbb{R}^{d}$.

Of course, we can define hyperbolic or spherical D-symbols in a similar way. An obvious necessary condition for a D-symbol to be good is that it locally "unfolds" correctly. This can be made precise by introducing appropriate substructures. For $(\mathcal{D}, M)$ a D-symbol with index set $I, D \in \mathcal{D}$ an arbitrary element and $J \subseteq I$, we define the Dsubsymbol generated by $D$ and $J$ to be the $\Sigma_{J}$-set $\mathcal{D}_{(D, J)}:=D \Sigma_{J}$, where $\Sigma_{J}$ is regarded as a subgroup of $\Sigma_{I}$ in the obvious way, together with the map $M_{(D, J)}:=\left.M\right|_{D \Sigma_{J} \times J \times J}$. Now a $d$-dimensional $\mathrm{D}$-symbol is called locally flat if all its $(d-1)$-dimensional subsymbols are spherical. Every good D-symbol must be locally flat.

Fortunately, there are simple effective criteria for determining whether a given twodimensional D-symbol is spherical, euclidean or hyperbolic [DH1], so, in particular, local flatness is easy to check in three-dimensional D-symbols. Namely, let $(\mathcal{D}, M)$ be a D-symbol with index set $I=\{i, j, l\}$. We define a function $k: \mathcal{D} \rightarrow \mathbb{Q}$ by

$$
k(D):=k_{i, j, l}(D):=\frac{1}{m_{i, j}(D)}+\frac{1}{m_{i, l}(D)}+\frac{1}{m_{j, l}(D)}-1
$$

and set $K(\mathcal{D}, M):=K_{i, j, l}(\mathcal{D}, M):=\sum_{D \in \mathcal{D}} k_{i, j, l}(D)$. The number $K(\mathcal{D}, M)$ is called the curvature of the D-symbol $(\mathcal{D}, M)$. It is closely related to the orbifold Euler characteristic in the sense of [Th2], which for manifolds coincides with the usual Euler characteristic. It is thus not surprising that the sign of $K(\mathcal{D}, M)$ distinguishes the curvature of the metric for a realization of $(\mathcal{D}, M)$. More precisely, we have:

Theorem 4. A two-dimensional D-symbol $(\mathcal{D}, M)$ is

- hyperbolic, iff $K(\mathcal{D}, M)<0$ holds,

- euclidean, iff $K(\mathcal{D}, M)=0$ holds,

- spherical, iff $K(\mathcal{D}, M)>0$ holds and, additionally, for all $i, j \in I$ and all $D \in \mathcal{D}$ the quantity

is a natural number and

$$
\frac{4}{v_{i, j}(D) \cdot K(\mathcal{D}, M)}
$$

- non-semi-good in all other cases. 
If $H$ is a group of automorphisms of a D-symbol ( $\mathcal{D}, M)$, then we denote by $(\mathcal{D}, M) / H$ the unique image $\left(\mathcal{D}^{\prime}, M^{\prime}\right)$ of $(\mathcal{D}, M)$ with $\mathcal{D}^{\prime}=\mathcal{D} / H$. In analogy to the topological setting, we find that for each subgroup $H$ of $\pi_{1}(\mathcal{D}, M)$, we have a D-symbol morphism from $(\widetilde{\mathcal{D}}, \tilde{M}) / H$ onto $(\mathcal{D}, M)$, which is an isomorphism exactly in the case $H=\pi_{1}(\mathcal{D}, M)$.

\section{Flatness in General Dimension}

In this section we discuss flatness criteria for finite D-symbols of arbitrary dimensions.

The topological realization of a finite D-symbol is compact, and there are only finitely many compact flat orbifolds in each dimension, as was shown by Bieberbach in his famous work [Bi]. Actually, Bieberbach proved that there are finitely many geometric types of crystallographic groups in each dimension. This result translates directly to orbifolds, see [Th3]. It would suffice therefore to compare the given symbol against each of these finitely many cases. Of course, this procedure is not the most practical in dimension 2 , extremely tedious if possible at all in dimension 3 and hopeless in higher dimensions. Fortunately, every flat $d$-orbifold is finitely covered by a $d$-dimensional torus $\mathbb{R}^{d} / \mathbb{Z}^{d}$. This follows from another one of Bieberbach's famous theorems, stating that each $d$ dimensional crystallographic space group $G$ (i.e., each co-compact, properly discontinuous group of euclidean isometries in dimension $d$ ) has a subgroup of finite index which is generated by $d$ linearly independent translations. Moreover, the subgroup $T(G)$ of all translations in a given group is of this type and is a normal and maximal abelian subgroup.

Based on this fact, we propose the following preliminary sketch of a flatness test:

Algorithm 5. Given a finite locally flat $d$-dimensional D-symbol $(\mathcal{D}, M)$.

1. Compute a presentation for $G:=\pi_{1}(\mathcal{D}, M)$.

2. For each positive integer $k$ in order, enumerate all subgroups of $G$ of index $k$.

3. Pick the first of these subgroups which is isomorphic to $\mathbb{Z}^{d}$ and call it $T$ (if none such exists, the D-symbol is not flat).

4. Compute the image $\left(\mathcal{D}^{\prime}, M^{\prime}\right):=(\widetilde{\mathcal{D}}, \widetilde{M}) / T$.

5. Determine whether $\left(\mathcal{D}^{\prime}, M^{\prime}\right)$ is unwound and its topological realization is a $d$-torus.

6. If not, continue at Step 3, this time picking the next unused subgroup.

If the maximal possible index of the translation subgroup is known, this procedure is finite, provided, of course, that each individual step can be carried out in finite time. If the test in Step 5 is negative for all subgroups picked in Step 3, then the given D-symbol is not flat, so in the general case, we only check a necessary condition for flatness. We will see later that in dimension 3 this condition is already sufficient.

Of course, in a certain sense, Step 3 cannot be carried out at all, because the isomorphism problem for finitely presented groups is undecidable. The following result helps:

Lemma 6. If $G$ is a space group of dimension $d$ and $N$ is a finite index subgroup of $G$, then $N$ is contained in $T(G)$ if and only if $N /[N, N]$ is isomorphic to $\mathbb{Z}^{d}$. In particular, if $G /[G, G] \cong \mathbb{Z}^{d}$ holds, then we have $G=T(G)$. 
Proof. If $N$ has finite index in $G$ and is contained in $T(G)$, then $N$ is a subgroup of finite index in $T(G)$, so it must of course be isomorphic to $\mathbb{Z}^{d}$.

To show the other implication, first note that any subgroup of finite index of a space group is again a space group, so we may restrict our attention to the case $N=G$.

If $G /[G, G]$ has rank $d$, then $(T(G) \cdot[G, G]) /[G, G]$, being a subgroup of finite index in $G /[G, G]$, also has rank $d$. However, then $(T(G) \cdot[G, G]) /[G, G] \cong T(G) /(T(G) \cap$ $[G, G])$ and $T(G)$ both have rank $d$, which forces $T(G) \cap[G, G]$ to be trivial.

Now if $g$ is an arbitrary element of $G$, and $h$ is an arbitrary element of $T(G)$, then $[g, h]$ is in $T(G)$, since $T(G)$ is a normal subgroup of $G$. By the above, then, we must have $[g, h]=1$, so every element of $G$ commutes with every element of $T(G)$. In particular, for any $g \in G$, the subgroup of $G$ generated by $T(G)$ and $g$, is abelian. However, since $T(G)$ is a maximal abelian subgroup of $G$, this implies $G=T(G)$.

Using this, we can replace Step 3 of Algorithm 5 by Step $3^{\prime}$ below and, moreover, dispose of Step 6, because if the original D-symbol is flat, then, using the homeomorphism $\alpha$ from Definition 3, $\alpha \circ \operatorname{TOP}(T) \circ \alpha^{-1}$ must be a translation subgroup of rank $d$ in the $d$-dimensional crystallographic space group $\alpha \circ \operatorname{TOP}(G) \circ \alpha^{-1}$.

Algorithm 7. Given a locally flat $d$-dimensional D-symbol $(\mathcal{D}, M)$.

1. Compute a presentation for $G:=\pi_{1}(\mathcal{D}, M)$.

2. For each positive integer $k$ in order, enumerate all subgroups of $G$ of index $k$.

$3^{\prime}$. Pick the first of these subgroups, call it $T$, such that $T /[T, T]$ is isomorphic to $\mathbb{Z}^{d}$.

4. Compute the image $\left(\mathcal{D}^{\prime}, M^{\prime}\right):=(\widetilde{\mathcal{D}}, \widetilde{M}) / T$.

5. Determine whether $\left(\mathcal{D}^{\prime}, M^{\prime}\right)$ is unwound and its topological realization is a $d$ torus.

In other words, we have:

Theorem 8. If $(\mathcal{D}, M)$ is a flat d-dimensional D-symbol with universal cover $(\widetilde{\mathcal{D}}, \widetilde{M})$ and fundamental group $G$, then there is a subgroup $T$ of finite index in $G$ such that $T /[T, T]$ is isomorphic to $\mathbb{Z}^{d}$ and for any such subgroup the image $(\widetilde{\mathcal{D}}, \widetilde{M}) / T$ is unwound and its topological realization is homeomorphic to a d-dimensional torus.

Now, as mentioned above, Step 1 can be performed by computing the presentation of Balke and Valverde, which utilizes the identity $\pi_{1}(\mathcal{D}, M) \cong \Sigma_{D} / \Lambda_{D}(\mathcal{D}, M)$, a Reidemeister-Schreier construction for $\Sigma_{D}$ and the fact that $\Lambda_{D}(\mathcal{D}, M)$ is normal in $\Sigma_{D}$. The last statement implies that a presentation for $\Sigma_{D} / \Lambda_{D}$ is obtained from one for $\Sigma_{D}$ simply by appending a list of generators for $\Lambda_{D}$ to the list of relators.

Given a finite presentation for $\pi:=\pi_{1}(\mathcal{D}, M)$, all subgroups up to some finite index $m$ can be found by the standard method of systematically generating all possible actions of $\pi$ on finite sets of sizes up to $m$, followed again by a Reidemeister-Schreier construction to obtain finite presentations (see [Ne]). If $N$ is such a subgroup, the rank and the torsion elements of $N /[N, N]$ can be determined by another standard method, namely by diagonalizing its relator matrix over $\mathbb{Z}$, after which the comparison against $\mathbb{Z}^{d}$ in Step $3^{\prime}$ becomes trivial. 
For Step 4, assume that a subgroup $H^{\prime}$ of $\pi$ is given via a permutation representation of $\pi$ on the set of cosets $\pi / H^{\prime}$. Let $H$ be its preimage with respect to the canonical projection $\Sigma_{D} \rightarrow \Sigma_{D} / \Lambda_{D} \cong \pi$. To simplify notation, we may identify $\pi / H^{\prime}$ with $\Sigma_{D} / H$ and $\widetilde{\mathcal{D}} / H^{\prime}$ with $\widetilde{\mathcal{D}} / H$, where $H$ now acts in the usual way as a subgroup of $\Sigma$. The following lemma tells us how to obtain $\widetilde{\mathcal{D}} / H$ from $\mathcal{D}$ and $\Sigma_{D} / H$ :

Lemma 9. Let $G$ be a group with e its neutral element, let $\varphi: S \rightarrow T$ be a $G$-map between transitive $G$-sets $S$ and $T$ and let $s \in S$ be arbitrarybut fix and set $t:=\varphi(s) \in T$. Let $r: T \rightarrow G$ be such as to satisfy $r(t)=e$ and $t \cdot r\left(t^{\prime}\right)=t^{\prime}$ for each $t^{\prime} \in T$. Finally, let $H$ be a subgroup of $G_{t}$ which contains $G_{s}$.

Then the $G$-set $S / H$ is isomorphic to $T \times G_{t} / H$ via

$$
\begin{aligned}
& S / H \rightarrow T \times G_{t} / H, \\
& s H g \mapsto\left(t g, H g \cdot r(t g)^{-1}\right),
\end{aligned}
$$

where $G$ acts on $T \times G_{t} / H$ by

$$
\left(t^{\prime}, H a\right) g:=\left(t^{\prime} g, H a \cdot r\left(t^{\prime}\right) \cdot g \cdot r\left(t^{\prime} g\right)^{-1}\right)
$$

for all $t^{\prime} \in T, a \in G_{t}$ and $g \in G$.

Proof. It is easy to see that the above defines a $G$-action on $T \times G_{t} / H$. Since $\left(t^{\prime}, H a\right)=$ $(t, H) a \cdot r\left(t^{\prime}\right)$ for all $t^{\prime} \in T$ and $a \in G_{t}$, the action is transitive. It suffices to show that the stabilizers of $(t, H)$ and $s H \in S / H$ are the same, i.e., that the stabilizer of $(t, H)$ is just $H$. Obviously for each $h \in H$, we have $(t, H) h=(t h, H h)=(t, H)$ because of $H \leq G_{t}$. If on the other hand, $(t, H) g=(t, H)$ holds for some $g \in G$, we have $t=t g$, thus $g \in G_{t}$, which in turn gives $r(t g)=e$, thus $H g=H$ and finally $g \in H$.

\section{Recognizing the 3-Torus}

There is no known general algorithm to test whether two three-dimensional manifolds are isomorphic. In spite of this, manifolds with sufficiently simple combinatorial representations have been successfully recognized and catalogued, as for example in [Li] and in $[\mathrm{Ma}]$.

Based on work by Haken [Ha2], it has been shown that for a special class of 3manifolds, now called Haken manifolds, the isomorphism problem can be solved algorithmically (see [He2]). A Haken manifold is a compact, oriented, irreducible 3-manifold which contains an incompressible surface of positive genus. Essentially, a 3-manifold $M$ is called irreducible if every 2-sphere properly embedded in $M$ bounds a 3-cell and an oriented surface $S$ of positive genus properly embedded in $M$ is called incompressible if every loop in $S$ which bounds a disk in $M$ bounds a disk in $S$.

Haken also describes a method to split a given PL-manifold $M$ into irreducible pieces [Ha1], i.e., to compute a collection of PL-manifolds $M_{1}, \ldots, M_{k}$ such that each $M_{i}$ is either irreducible or isomorphic to $S^{2} \times S^{1}$ (the only reducible 3-manifold which is no non-trivial connected sum) and such that $M$ is just the connected sum $M_{1} \# \ldots \# M_{k}$. The connected sum of two manifolds is obtained by removing a cell from the interior of 
each and gluing together the resulting manifolds along the boundaries of these cells. It has been shown that for oriented manifolds the result does not depend on the particular gluing and, moreover, that every compact oriented 3-manifold different from the 3sphere is uniquely decomposable with respect to connected sums into a collection of so-called prime manifolds. A manifold different from $S^{3}$ is called prime unless it is a connected sum of two manifolds both different from $S^{3}$, i.e., if it is either incompressible or isomorphic to $S^{2} \times S^{1}$ (see [He1]).

Unfortunately, there can be copies of $S^{3}$ among the pieces obtained by Haken's method, i.e., the collection of prime manifolds obtained may not be minimal. As obviously the 3-sphere does not contain an incompressible surface, Haken's method is not applicable to it. An algorithm to recognize the 3-sphere based on so-called almost normal surfaces was recently presented by Rubinstein $[\mathrm{Ru}]$ and elaborated on by Thompson [Th1]. We refer to it as the Rubinstein-Thompson method.

Theorem 10. There is an algorithm to check whether a given PL-manifold is homeomorphic to the 3-torus $T^{3}:=S^{1} \times S^{1} \times S^{1}$.

Proof. Let $M$ be a PL-manifold given in a suitable way, e.g., as a triangulation. Denote by $H$ the first integral homology group of $M$. If $M$ is the 3-torus, then $H$ must be isomorphic to $\mathbb{Z}^{3}$ as a group. This can be checked in finite time, and in the following, we assume that it is the case.

Using Haken's method, split $M$ into a collection of prime manifolds $M_{1}, \ldots, M_{k}$ with homology groups $H_{1}, \ldots, H_{k}$ such that $M$ is isomorphic to $M_{1} \# \ldots \# M_{k}$. Necessarily then, we have $H=H_{1} \times \cdots \times H_{k}$. The 3-torus is prime, so $M$ is isomorphic to $T^{3}$ if and only if exactly one of the pieces is, while all other pieces are isomorphic to $S^{3}$. We may assume without loss of generality that $H_{1}=\mathbb{Z}^{3}$ and $H_{i}=1$ for $i=2, \ldots, k$ hold.

Every oriented 3-manifold with infinite first homology contains an incompressible surface of positive genus (see [He1]), thus, as $H_{1}$ is irreducible by construction, it must be Haken. We can therefore apply the isomorphism test for Haken manifolds.

On each of the remaining pieces, the Rubinstein-Thompson test can be applied to check whether all of them are isomorphic to $S^{3}$.

\section{Flatness and D-Symbol Morphisms}

Theorem 8 provides a necessary flatness condition for D-symbols of arbitrary dimensions. Roughly, it says that a cover with a certain property must exist and that the cover must be flat. In general, any cover of a flat D-symbol is flat, because its fundamental group will be a subgroup of the original symbol's. If the reverse was true as well, this would imply the sufficiency of the condition from Theorem 8 .

In dimensions 2 and 3 this is indeed the case. In effect, every periodic tiling of $\mathbb{R}^{2}$ or $\mathbb{R}^{3}$ can be realized with its full combinatorial symmetry. In dimension 2 this even holds for spherical and hyperbolic tilings and is an easy consequence of the geometric classification theorem for two-dimensional D-symbols [DH1], [Hu1] (see also $[\mathrm{Sc}])$. 
In dimension 3 the result follows from an important theorem in geometric topology [MS]:

Theorem 11 [MS]. Let $M$ be a 3-manifold with a geometric structure modelled on one of $H^{2} \times \mathbb{R}, \mathrm{SL}_{2} \mathbb{R}, N i l, \mathbb{E}^{3}$ or Sol. Then, up to conjugacy, any smooth finite group action on $M$ preserves the geometric structure on $M$.

By standard arguments, the conclusion also holds for group actions which fix a (pseudo-)triangulation of the given manifold, so in particular, it can be applied to the case where $G$ is a group of D-symbol automorphisms acting on $\mathcal{D}, \operatorname{TOP}(\mathcal{D})$ allowing for a geometric structure of one of the five listed types. The theorem then implies that the given metric can be adjusted via a self-homeomorphism of $\operatorname{TOP}(\mathcal{D})$ to one on which $\operatorname{TOP}(G)$ acts by isometries.

Theorem 12. If $(\mathcal{D}, M)$ and $\left(\mathcal{D}^{\prime}, M^{\prime}\right)$ are finite three-dimensional $D$-symbols and there is a covering $\varphi:(\mathcal{D}, M) \rightarrow\left(\mathcal{D}^{\prime}, M^{\prime}\right)$, then $(\mathcal{D}, M)$ is flat if and only if $\left(D^{\prime}, M^{\prime}\right)$ is flat.

Proof. If $\left(\mathcal{D}^{\prime}, M^{\prime}\right)$ is flat, then trivially this holds for $(\mathcal{D}, M)$. In the following, we assume that $(\mathcal{D}, M)$ is flat and show that $\left(\mathcal{D}^{\prime}, M^{\prime}\right)$ is as well.

Let $G:=\pi_{1}(\mathcal{D}, M)$ and $G^{\prime}:=\pi_{1}\left(\mathcal{D}^{\prime}, M^{\prime}\right)$ be the fundamental groups of $(\mathcal{D}, M)$ and $\left(\mathcal{D}^{\prime}, M^{\prime}\right)$, respectively. We may assume without loss of generality that $(\mathcal{D}, M)$ is unwound, thus $\operatorname{TOP}(\mathcal{D})$ is a manifold, and that $G$ is normal in $G^{\prime}$. Indeed, any finite cover of $(\mathcal{D}, M)$ will be flat. By Theorem $8,(\mathcal{D}, M)$ has an unwound finite cover, so we assume that $(\mathcal{D}, M)$ is unwound. If $G$ is not normal in $G^{\prime}$, then we replace $(\mathcal{D}, M)$ by the finite cover corresponding to the core of $G$ in $G^{\prime}$.

Because $G$ is normal in $G^{\prime}$, the action of $G^{\prime}$ on $(\widetilde{\mathcal{D}}, \widetilde{M})$ induces an action on $(\mathcal{D}, M)$. Now since $(\mathcal{D}, M)$ is flat, $\operatorname{TOP}(\mathcal{D})$ is homeomorphic to the orbit space of $\mathbb{E}^{3}$ by an isometric $G$-action. In effect, $G^{\prime}$ acts on the flat manifold $\operatorname{TOP}(\mathcal{D})$ by homeomorphisms, fixing the pseudo-triangulation induced by the $\Sigma$-set structure. The finite index subgroup $G$ of $G^{\prime}$ acts trivially, so the $G^{\prime}$-action is effectively only a finite one. By Theorem 11, we may assume that $G^{\prime}$ preserves the induced flat metric on $\operatorname{TOP}(\mathcal{D})$.

Lifting to the universal cover, we finally obtain the desired isometric action of $G^{\prime}$ on $\mathbb{E}^{3}$.

Theorem 12 has two important consequences. Firstly, it tells us that in dimension 3 the necessary condition from Theorem 8 is also sufficient. Because of its importance, we state this as a separate theorem.

Theorem 13. Let $(\mathcal{D}, M)$ be a three-dimensional D-symbol with universal cover $(\widetilde{\mathcal{D}}, \widetilde{M})$ and fundamental group $G$.

If $(\mathcal{D}, M)$ is flat, then there is a subgroup $T$ of index at most 48 in $G$ such that $T /[T, T]$ is isomorphic to $\mathbb{Z}^{d}$.

If, on the other hand, $T$ is such a subgroup, then $(\mathcal{D}, M)$ is flat if and only if the image $(\widetilde{\mathcal{D}}, \widetilde{M}) / T$ is unwound and its topological realization is homeomorphic to a three-dimensional torus. 
Corollary 14. There is an algorithm to check whether a given three-dimensional Dsymbol is flat.

Proof. This follows directly from Theorems 10 and 13.

Our main result follows:

Theorem 15. For each natural number $n$, the collection of all (isomorphism classes of) D-symbols of size not greater than $n$ corresponding to periodic tilings of threedimensional euclidean space can be computed in finite time.

Proof. Obviously, there are up to isomorphism only finitely many $\Sigma$-sets of each given size. As the branching limits in flat D-symbols are subject to the crystallographic restriction, i.e., can only be $1,2,3,4$ or 6 , there are only finitely many D-symbols of each given size which have to be checked for flatness.

Strictly speaking, Theorem 15 does not say that we can construct an actual representative of each equivalence class of three-dimensional euclidean tilings. All we know is that such a tiling exists. The main reason for this is that we have used Theorem 11 as a "black box", a mere existence result. However, the proof given by Meeks and Scott actually is constructive. It does not seem unreasonable therefore to believe that Algorithm 7 can be extended to construct a tiling for each D-symbol found to be flat. A detailed description of such an extended algorithm however is beyond the scope of this article. In applications so far, heuristic methods have been used which were successful in most, but not all, cases.

Secondly, it suffices to test flatness for only one representative of each class of Dsymbols which are connected by some chain of coverings. We call a D-symbol minimal if there is no non-trivial D-symbol morphism from it onto any other D-symbol. It can be shown that to each D-symbol $(\mathcal{D}, M)$ there is a unique minimal image, called the universal image, such that every other image of $(\mathcal{D}, M)$ also maps onto it. Therefore, in classifications of three-dimensional tilings, we usually restrict ourselves to mimimal D-symbols. These, if flat, represent tilings which are maximally symmetric in their respective topological families.

\section{Speeding up the Subgroup Search}

The complexity of subgroup enumeration, as required in Step 2 of Algorithm 7, is estimated to grow at least exponentially with the index. In dimension 3 already, this index can be as large as 48 . We discuss two strategies to avoid enumerating large index subgroups.

The first strategy is applicable whenever sufficiently much is known on the structure of possible point groups. Every euclidean isometry can be described as the product of an orthogonal linear map and a translation. The map which takes each isometry to its linear component is a group homomorphism. For a given crystallographic space group, 
the image is called its point group and (obviously) is isomorphic to the quotient of the original group by its translation subgroup. The possible point groups are exactly those finite subgroups of the full orthogonal group which fulfil the so-called crystallographic restriction, i.e, do not contain non-trivial rotations of orders other than $2,3,4$ or 6 . In dimension 3 there are, up to conjugation in $O_{3}(\mathbb{R})$, precisely 23 such groups, all of which are isomorphic to some group of the form $H, H \times C_{2}$ or $H \times C_{2} \times C_{2}$, where $H$ is a subgroup of $S_{4}$ and $C_{2}$ is the group with two elements.

All candidates for translation subgroups of a given D-symbol fundamental group are thus found by enumerating kernels of homomorphisms into $S_{4}$ and intersecting these with up to two subgroups of index 2 . To enumerate the kernels, all conjugacy classes of subgroups of index up to 4 are constructed. For each of these subgroups its core, i.e., the largest normal subgroup of the original group contained in it, is determined, again by intersection methods [NF], [De2].

The second strategy is based on geometric features of the given D-symbol and its topological realization. It works by successively computing subgroups of small index and their corresponding covers and in each step choosing exactly one which is in some sense simpler than the previous symbol. In [De2] it is shown that for each three-dimensional euclidean D-symbol a subgroup of the translation group can be found by successively constructing covers of index 3 at most twice and of index 2 at most five times.

As a first step, it is easy to see that, in any dimension, a D-symbol is oriented if and only if the symmetry group associated to it acts in an orientation preserving way. For a nonoriented D-symbol, there is a canonical oriented two-sheeted cover which corresponds to the orientation-preserving subgroup. Succeeding steps proceed by "eliminating" certain types of singular points from the corresponding orbifold until the resulting cover is a manifold and then its first integral homology is used as an invariant. It is conceivable that similar methods will be useful for other metrics or higher dimensions.

In practice, combinations of the two strategies have been most successful. In dimension 3 for example, point groups of the form $H \times C_{2} \times C_{2}$ are never orientation preserving. In [DH3] we describe certain invariants which distinguish the 219 crystallographic space groups in dimension 3 . These can be used to avoid the subgroup search completely in some cases, while in others, advance knowledge of the (potential) point group will allow a restricted search.

\section{The 3-Torus Recognition Problem in Practice}

The key to practical manifold recognition is to simplify a given representation as far as possible, thus obtaining either a manifold homeomorphic to the original one, or else a collection of connected summands of the original manifold. If the resulting representations are small enough, they can be looked up in a catalogue. Otherwise, more sophisticated methods can be applied. Algorithms based on the enumeration of embedded surfaces as referred to in Section 4 tend to have extremely high computational complexity. Thus almost every effort to reduce the size of their input data should be worthwhile.

The strategy sketched below for the torus-recognition problem has, in spite of its obvious limitations, proved successful in many applications [De2], [DH4], [DDH ${ }^{+}$, [DH5]. In this and the following section we only consider unwound D-symbols, so we 
restrict our attention to the underlying $\Sigma$-sets. Moreover, we always assume that these are three-dimensional, oriented and locally flat, thus their topological realizations are oriented three-dimensional manifolds (see [LM]).

Algorithm 16. Let $\mathcal{D}$ be an oriented locally flat $\Sigma$-set with fundamental group $G$ such that $G /[G, G] \cong \mathbb{Z}^{3}$. The algorithm searches for criteria telling whether $\operatorname{TOP}(\mathcal{D})$ is homeomorphic to the three-dimensional torus $T^{3}=S^{1} \times S^{1} \times S^{1}$. Possible outcomes: "yes", "no" or "unsure" (the latter meaning that no criterion was found which would allow a decision).

1. Try to prove inequality by group-theoretic invariants. In detail:

(a) Compute the number $k$ of conjugacy classes of subgroups of $G$ with index up to 4 . If $k$ is different from 56 (the corresponding number for $\mathbb{Z}^{3}$ ), return "no".

(b) Determine whether the (abelian) quotient $[G, G] /[G,[G, G]]$ is trivial. Otherwise, return "no". We use a method described in [Ni] to compute this quotient.

2. Apply a simplification procedure to obtain either

(a) a simpler $\Sigma$-set $\mathcal{D}^{\prime}$ such that $\operatorname{TOP}\left(\mathcal{D}^{\prime}\right)$ is homeomorphic to $\operatorname{TOP}(\mathcal{D})$ or

(b) a proof that $\operatorname{TOP}(\mathcal{D})$ is not a 3-torus (return "no" in this case).

3. Check whether $\mathcal{D}$ is contained (up to an isomorphism) in a short list of simple representations of the 3-torus. If so, return "yes", otherwise, return "unsure".

\section{Simplification of $\Sigma$-Sets}

We start this section by describing a rather general type of operation on $\Sigma$-sets due to Dress (see [Fr]). Let $\mathcal{D}$ be a finite $\Sigma$-set with index set $I$. For some $i \in I$, let $\mathcal{A}$ be a $\sigma_{i}$-invariant subset of $\mathcal{D}$, thus $D \sigma_{i} \in \mathcal{A}$ for all $D \in \mathcal{A}$. Define a new $\Sigma$-set $\mathcal{D}^{\prime}$ as the set $\mathcal{D}-\mathcal{A}$ together with the $\Sigma$-action $\circ: \mathcal{D}^{\prime} \times \Sigma \rightarrow \mathcal{D}^{\prime}$ given by $D \circ \sigma_{j}:=D \sigma_{j}\left(\sigma_{i} \sigma_{j}\right)^{r}$ where $r:=\min \left\{n \in \mathbb{N}_{0} \mid D \sigma_{j}\left(\sigma_{i} \sigma_{j}\right)^{n} \in \mathcal{D}^{\prime}\right\}$, for all $D \in \mathcal{D}^{\prime}$ and $j \in I$. It is not hard to show that $r$ is always well-defined and that $\left(D \circ \sigma_{j}\right) \circ \sigma_{j}=D$ holds for all relevant $D$ and $j$, so $\circ$ is indeed a $\Sigma$-action. We call the operation of removing a $\sigma_{i}$-invariant subset $\mathcal{A}$ and redefining the $\Sigma$-action as above an $i$-reduction and denote the reduced $\Sigma$-set $\mathcal{D}^{\prime}$ by $\mathcal{D} \backslash{ }_{i} \mathcal{A}$. We sometimes abuse this notation to refer to the specific reduction itself.

It is easy to see that for disjoint $i$-invariant subsets $\mathcal{A}$ and $\mathcal{B}$, we have $\left(\mathcal{D} \backslash{ }_{i} \mathcal{A}\right) \backslash{ }_{i} \mathcal{B}=$ $\mathcal{D} \backslash{ }_{i}(\mathcal{A} \cup \mathcal{B})$. To study the behaviour of $i$-reductions in general, we consider elementary $i$-reductions, where $\mathcal{A}$ is a single $\sigma_{i}$-orbit, thus $\mathcal{A}=\left\{D, D \sigma_{i}\right\}$ for some $D \in \mathcal{D}$. In the following we assume that $\Sigma$ is loopless, so every $\sigma_{i}$-orbit has exactly two elements. Define $J:=\left\{j \in I \mid D \sigma_{j}=D \sigma_{i}\right\}$. There are essentially three cases:

1. In the special case $J=I$, if $\mathcal{D}$ is transitive, $\operatorname{TOP}(\mathcal{D})$ is homeomorphic to an $n$-sphere and the result of the $i$-reduction is empty. Otherwise, a connected component of $\operatorname{TOP}(\mathcal{D})$ homeomorphic to an $n$-sphere is removed.

2. If $D \Sigma_{I-J}$ and $D \sigma_{i} \Sigma_{I-J}$ are disjoint, then the effect of the reduction on the topological realization of $\mathcal{D}$ is that of removing the interior of an embedded closed ball and identifying opposite hemispheres of its boundary. Consequently, $M^{\prime}:=\operatorname{TOP}\left(\mathcal{D} \backslash_{i}\left\{D, D \sigma_{i}\right\}\right)$ is homeomorphic to $M:=\operatorname{TOP}(\mathcal{D})$. This kind of operation has been studied in the theory of GEMs, where it is called a fusion, see [LM]. 
3. If $D \Sigma_{I-J}=D \sigma_{i} \Sigma_{I-J}$ holds, then $M^{\prime}$ is in general not homeomorphic to $M$. The effect of the $i$-reduction varies. In dimension $2 \operatorname{TOP}\left(\mathcal{D} \backslash_{i}\left\{D, D \sigma_{i}\right\}\right)$ is derived from $M$ by cutting along an embedded circle and capping off the two boundaries by disks.

In dimension 3 there are four subcases depending on the set $\mathcal{K}:=\{\emptyset \neq K \subset$ $\left.I-J \mid D \Sigma_{K} \neq D \sigma_{i} \Sigma_{K}\right\}$. We define a Graph $\mathcal{G}=\mathcal{G}(\mathcal{K}):=(\mathcal{K}, V(\mathcal{K}))$ where $V(\mathcal{K}):=\{\{A, B\} \in \mathcal{K} \times \mathcal{K} \mid A \subset B\}$. Since each $K \in \mathcal{K}$ has either one or two elements, each connected component of $\mathcal{G}$ is either a chain or a cycle. Let $m$ be the number of connected components.

(a) If $m=1$ and $\mathcal{G}$ is a cycle, necessarily \# $J=1$ and the intersection $S$ of the simplices $|D|$ and $\left|D \sigma_{i}\right|$ corresponding to $D$ and $D \sigma_{i}$ in $M$ consists of their common $i$-face and the opposite vertex. Consequently, $M^{\prime}$ is derived from $M$ by removing a properly embedded solid torus and gluing in a cone on its boundary. We call this operation a torus capping for the moment.

(b) If $m=1$ and $\mathcal{G}$ is a chain, $M^{\prime}$ is homeomorphic to $M$.

(c) If $m=2$, then $M^{\prime}$ is derived from $M$ by cutting along a properly embedded 2sphere contained in $|D| \cup\left|D \sigma_{i}\right|$ and capping off the resulting boundary spheres by disks. We call this a cut. If the result $M^{\prime}$ has two connected components, then $M$ is homeomorphic to the connected sum of the components of $M^{\prime}$ and we call the operation a decomposition. Otherwise, we can express $M$ as the connected sum of $M^{\prime}$ with $S^{2} \times S^{1}$. The operation of removing an $S^{2} \times S^{1}$ summand is called a three-dimensional handle reduction.

(d) The case $m=3$ can be described as a sequence of two cuts, each of which is either a decomposition or a handle reduction.

We call an elementary $i$-reduction on a three-dimensional $\Sigma$-set admissible if it is not of type 3(a), i.e., if it is not a torus capping and its result is not empty. We call a general $i$-reduction admissible if it can be decomposed into a series of admissible elementary $i$-reductions.

The following are immediate consequences:

Theorem 17. If $\mathcal{D}$ is a transitive, oriented, locally flat three-dimensional $\Sigma$-set with at least four elements and $\mathcal{D}^{\prime}$ is derived from $\mathcal{D}$ by a sequence of admissible elementary $i$ reductions, then $\operatorname{TOP}(\mathcal{D})$ can be uniquely reconstructed given $\operatorname{TOP}\left(\mathcal{D}^{\prime}\right)$ and the number $z$ of handle reductions performed, namely as the connected sum of all the connected components of $\operatorname{TOP}\left(\mathcal{D}^{\prime}\right)$ and $z$ copies of $S^{2} \times S^{1}$.

Corollary 18. Given $\mathcal{D}, \mathcal{D}^{\prime}$ and $z$ as above, $\operatorname{TOP}(\mathcal{D})$ is a 3-torus if and only if exactly one of the connected components of $\mathrm{TOP}\left(\mathcal{D}^{\prime}\right)$ is a 3-torus, all others are 3-spheres and $z=0$.

Proof. This is obvious, because the 3-torus is prime.

Corollary 19. If $\mathcal{D}$ is a three-dimensional $\Sigma$-set on which a handle-reduction can be performed, then $\operatorname{TOP}(\mathcal{D})$ is not a 3-torus. 
Corollary 20. If $\mathcal{D}$ is an oriented, locally flat three-dimensional $\Sigma$-set with at least four elements and $\mathcal{D}^{\prime}$ is derived from $\mathcal{D}$ by a sequence of admissible elementary $i$-reductions, then the number of handle reductions in any other sequence of admissible elementary $i$-reductions which produce $\mathcal{D}^{\prime}$ is the same.

Moreover, we have

Lemma 21. If $D^{\prime}=D \sigma_{i}=D \sigma_{j}$ holds for some element $D$ of a three-dimensional $\Sigma$-set $\mathcal{D}$ and $i \neq j$, then $\mathcal{D} \backslash{ }_{i}\left\{D, D \sigma_{i}\right\}$ is an admissible reduction.

It was shown in [FG] that a pair of GEMs of any dimension $d$ represent homeomorphic manifolds if and only if one can be transformed into the other by a sequence of fusions and their inverses. This result applies directly to unwound, loopless, locally flat $\Sigma$-sets. Unfortunately, no general method is known to compute upper bounds for the minimal lengths of such sequences. The isomorphism problem for manifolds can therefore not be solved by systematically applying all sequences of fusions and inverse fusions on a given $\Sigma$-set.

Anyway, it is straightforward to detect admissible elementary $i$-reductions in a $\Sigma$-set, construct the reduced symbol and-in dimension 3-determine the number of $S^{2} \times S^{1}$ summands which were removed in the process. This process can then be repeated until there are no further admissible $i$-reductions. However, for a practically useful algorithm, such a strategy is not sufficient.

We now restrict our attention to dimension 3 once again.

A starting point for Lins' approach [Li] is the observation that certain operations which do not change the size of the $\Sigma$-set may lead to a situation where an elementary reduction can be applied. One such operation is the so-called p-pair switching: given $D, D^{\prime} \in \mathcal{D}$ such that $D^{\prime} \notin\left\{D, D \sigma_{i}\right\}$ but $D^{\prime} \in D \Sigma_{i, j} \cap D \Sigma_{i, k}$ for some triplet $\{i, j, k\} \subset I$, where $\mathcal{D}$ is oriented and $d(D) \neq d\left(D^{\prime}\right)$, the $\sigma_{i}$-operation can be changed to connect $D$ with $D^{\prime}$ and the former $D \sigma_{i}$ with the former $D^{\prime} \sigma_{i}$. If $\mathcal{D}$ is locally flat, this increases the number of $\Sigma_{i, j, k}$-orbits, thus allowing for an admissible $l$-reduction in the new symbol, where $\{i, j, k, l\}=I$.

Another set of operations deals with configurations of three or more quadrangles. A quadrangle in a $\Sigma$-set is a loopless $\Sigma_{i, j}$-orbit of size 4 for some $i, j \in I$. Certain assemblies of quadrangles allow for local changes, so-called TS-moves, which do not affect the topology. Lins' algorithm constructs the (finite) list of all $\Sigma$-sets which can be derived from a given one, say $\mathcal{D}$, by applying all possible sequences of TS-moves and simplifies each of these as far as possible by sequences of elementary $i$-reduction. If any of the results is smaller than $\mathcal{D}$, the simplification process continues with it instead of $\mathcal{D}$. This is called the TS-algorithm. The reason for its usefulness lies in the fact that whenever four quadrangles contain a common element, the $\Sigma$-set in question can be simplified. Moreover, by standard arguments based on the Euler characteristic, a loopless, locally flat, three-dimensional $\Sigma$-set without $\Sigma_{i, j}$-orbits of size 2 must contain at least 12 quadrangles.

Going even further, Lins introduces an operation, called the $U$-move, which increases the size of a $\Sigma$-set, but introduces many quadrangles. Depending on the number $k$ of 
$U$-moves which are allowed before applying the TS-algorithm, this gives rises to an infinite number of so-called $U_{k}$-algorithms.

In contrast, D-symbols and $\Sigma$-sets derived from tilings have a special property which we call regularity. A $d$-dimensional D-symbol $(\mathcal{D}, M)$ is regular if $m_{i j}(D)=2$ holds for all $D \in \mathcal{D}$ and all pairs of indices satisfying $|i-j|>1$. A $d$-dimensional $\Sigma$-set $\mathcal{D}$ is regular if for indices $i, j$ satisfying $|i-j|>1$, every $\Sigma_{i, j}$-orbit is a quadrangle. The D-symbol of a tiling is always regular. If $(\mathcal{D}, M)$ is regular, unwound and loopless, then $\mathcal{D}$ is regular.

The simplification process we use in our applications to tiling theory is designed to retain-and make use of-regularity in each step. The basic operation is a nonelementary $i$-reduction which we call a collapse. Consider $\{i, j, k\} \subset I$ and $D \in \mathcal{D}$ such that every $\Sigma_{i, j}$ - and $\Sigma_{i, k}$-orbit in $D \Sigma_{i, j, k}$ is a quadrangle. In other words, $D^{\prime}\left(\sigma_{i} \sigma_{j}\right)^{2}=$ $D^{\prime}\left(\sigma_{i} \sigma_{k}\right)^{2}=D^{\prime}$ for every $D^{\prime} \in D \Sigma_{i, j, k}$. If $\mathcal{D}$ is oriented, $\mathcal{D} \backslash_{i}\left\{D, D \sigma_{i}\right\}$ is admissible and $\mathcal{D}-D \Sigma_{i, j, k}$ is non-empty, then $\mathcal{D} \backslash_{i}\left(D \Sigma_{i, j, k}\right)$ is admissible. The proof is a simple induction based on Lemma 21 . We call $\mathcal{D} \backslash_{i}\left(D \Sigma_{i, j, k}\right)$ an $(i ; j, k)$-collapse. Obviously, collapses preserve regularity.

Any admissible 0 -reduction on a regular, oriented $\Sigma$-set leads to an admissible $(0 ; 2,3)$-collapse. Likewise, any admissible 3 -reduction leads to an admissible $(3 ; 0,1)$ collapse. These two types of collapses can be interpreted as the removal of walls (2-faces) and the shrinking of edges in the corresponding tiling. Sometimes 1- or 2-reductions lead to admissible collapses as well, which can be interpreted as the removal of certain degeneracies in the tiling. As in Lins' algorithm, we make use of a limited set of operations which do not decrease the size of the $\Sigma$-set. These are expressed as combinations of collapses and their inverses. Unlike Lins, we are able to introduce a slightly more complicated complexity measurement such that each step of our algorithm decreases the complexity of the $\Sigma$-set, thus by showing that this complexity can only be decreased a finite number of times we establish that the algorithm eventually stops.

Details will appear in a forthcoming paper, in which we will also address reduction methods for general, i.e., possibly not unwound, D-symbols.

\section{An Example}

To illustrate the ideas of this text, we consider the classification of flat regular D-symbols with a common underlying $\Sigma_{0,1,2,3}$-set $\mathcal{D}_{0}$ shown in Fig. 1 . In our figures the $\sigma_{i}$ are represented by edges of different styles, as indicated in the legend in Fig. 1.

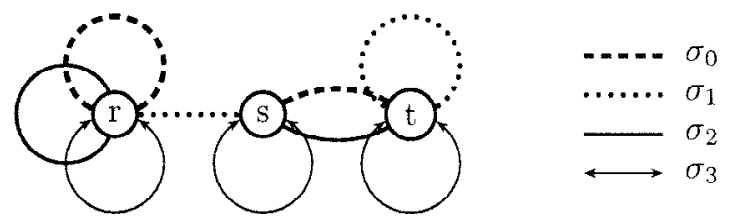

Fig. 1. The $\Sigma$-set $\mathcal{D}_{0}$. 


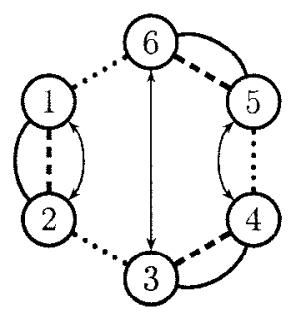

Fig. 2. An oriented $\Sigma$-set $\mathcal{D}$ mapping onto $\mathcal{D}_{0}$.

Figure 2 shows a twofold oriented $\Sigma$-preimage $\mathcal{D}$ of $\mathcal{D}_{0}$. There is a unique $\Sigma$-map $\rho: \mathcal{D} \rightarrow \mathcal{D}_{0}$, namely the one which maps $1 \in \mathcal{D}$ to $r \in \mathcal{D}_{0}$.

If $(\mathcal{D}, M)$ is a regular D-symbol based upon this $\Sigma$-set, then the function $M: \mathcal{D} \rightarrow \mathbb{N}$ is completely determined by the four values $a:=m_{0,1}(1), b:=m_{1,2}(1), c:=m_{2,3}(1)$ and $d:=m_{2,3}(3)$. Both $a$ and $b$ must be multiples of 3 , while $d$ must be even and $c$ may be an arbitrary natural number. In particular, the $\Sigma$-map $\rho$ will extend to a D-symbol covering for every possible choice of these numbers, and we can restrict our attention to $\mathcal{D}$. Our first goal is to find all possible choices of $a, b, c$ and $d$ such that the resulting D-symbol is locally flat.

We start by considering the single $\Sigma_{0,1,2}$-orbit in $\mathcal{D}$. By regularity, we have $m_{0,2}(D)=$ 2 for each $D \in \mathcal{D}$, which implies $k:=k_{0,1,2}(D)=1 / a+1 / b-\frac{1}{2}$. Thus, the subsymbol obtained by making a particular choice for $a$ and $b$ will have curvature $6 k$, which is positive only for $a=b=3$. In this case the second condition from Theorem 4 holds as well, so the resulting subsymbol is spherical.

Because $m_{0,3}(D)=m_{1,3}(D)=2$, we have $k_{0,1,3}(D)=1 / a>0$ for each $D \in$ $\mathcal{D}$, thus the $\Sigma_{0,1,3}$-subsymbol is always spherical. Likewise, all $\Sigma_{0,2,3}$-subsymbols are spherical regardless of the choice of $c$ and $d$ (this holds for all regular D-symbols) and it remains to consider the single $\Sigma_{1,2,3}$-orbit. Here, we have

$$
\begin{aligned}
K:=K\left(\mathcal{D}_{(1,\{1,2,3\}}, M_{(1,\{1,2,3\})}\right) & =2 k_{1,2,3}(1)+4 k_{1,2,3}(3) \\
& =2\left(\frac{1}{3}+\frac{1}{c}-\frac{1}{2}\right)+4\left(\frac{1}{3}+\frac{1}{d}-\frac{1}{2}\right) \\
& =\frac{2}{c}+\frac{4}{d}-1,
\end{aligned}
$$

thus $K>0$ implies $2 / c+4 / d>1$. We discard solutions with $c$ or $d$ smaller than 3 , as these would correspond to "degenerate" tilings with certain edges incident to only one or two tiles. Furthermore, by the crystallographic restriction, in a flat D-symbol we must have $v_{i, j}(D) \in\{1,2,3,4,6\}$ for all $i, j \in I$ and $D \in \mathcal{D}$. There remain six possible combinations of values for $c$ and $d$, namely $(3,4),(3,6),(3,8),(4,4),(4,6)$ and $(6,4)$. Each of these is admissible in the sense that it induces a spherical $\Sigma_{1,2,3}$-subsymbol. The corresponding complete D-symbols will be denoted by $\left(\mathcal{D}, M_{3,4}\right),\left(\mathcal{D}, M_{3,6}\right)$ and so on.

From the combination of branching numbers in each individual two-dimensional subsymbol, one can determine the geometric type of a corresponding vertex-, edge-, faceor tile-stabilizer in a flat realization. As each of these stabilizers must be contained in 
the point group, we may find that certain point groups are impossible. Only orientationpreserving groups have to be considered, as $\mathcal{D}$ is oriented. Here, it turns out that for the cases $(3,6),(3,8)$ and $(4,6)$, the point group must be the group of orientationpreserving symmetries of the octahedron, labelled $O$ in Schönfliess notation and 432 in crystallographic notation. This group is abstractly isomorphic to the symmetric group $S_{4}$. In the cases $(3,8)$ and $(4,6)$, this is obvious from the fact that there are rotations of both orders 3 and 4 , whereas in case $\left(3,6\right.$ ), both the groups $T$ (or 23) and $D_{3}$ (or 322 ) occur as stabilizers, which are conjugate to common subgroups only in 432 . In the case $(6,4)$, the point group must clearly be $D_{6}$ (or 622), whereas in the case $(4,4)$, the point group cannot be one of $T$ or $O$ due to the fact that a three-dimensional irreducible crystallographic space group must contain a rotation of order 3, which leaves $D_{4}$ (or $422)$ as the only possible point group. Only in the case $(3,4)$ is the stabilizer information not sufficient to uniquely determine the geometric type of the point group.

Next we check whether any of the six fundamental groups in question contains a normal subgroup with factor group isomorphic to $S_{4}$. We start by enumerating arbitrary homomorphisms into $S_{4}$, i.e., actions of these groups on a four-element set and then pick out the surjective ones. Our computer implementation constructs explicit presentations of the fundamental groups in terms of abstract generators and relators. It is usually more efficient to work on these, but in view of Lemma 9, we can equivalently construct all transitive fourfold coverings of the given D-symbol and read off the required information as follows.

We assign labels from the set $\{A, B, C, D\} \times \mathcal{D}$ to the elements of the covering Dsymbol. These are abbreviated to A1, A2, . in the figures. The first component of each label corresponds to an element of the set $\{A, B, C, D\}$, while the second component determines the image in the $\Sigma$-set $\mathcal{D}$. Given a $\Sigma$-map onto $\mathcal{D}$ which matches these labels, we can assign to each pair $(D, i) \in \mathcal{D} \times\{0,1,2,3\}$ a permution $\alpha$ of the set $\{A, B, C, D\}$ by defining $\alpha(X):=Y$ precisely if $(X, D) \sigma_{i}=\left(Y, D \sigma_{i}\right)$ holds. The pairs $(D, i)$ can be identified with generators of the fundamental group $G:=\pi_{1}(\mathcal{D}, M)$. The corresponding map $G \rightarrow S_{4}$ is well-defined if and only if the $\Sigma$-map induces a covering of D-symbols. This methods works because the fundamental group of the cover will be just the stabilizer of some element with respect to the action on $\{A, B, C, D\}$.

If $\varphi:\left(\mathcal{D}^{\prime}, M^{\prime}\right) \rightarrow(\mathcal{D}, M)$ is a covering, then its restriction to any $\Sigma_{J}$-subsymbol must induce a covering of $\Sigma_{J}$-symbols. We therefore start by looking at possible two-, three- or fourfold covers of the $\Sigma_{0,1,2}$-subsymbol common to all our candidates. Because $m_{0,1} \equiv$ $m_{1,2} \equiv 3$, both $r_{0,1}(X, D)$ and $r_{1,2}(X, D)$ must be 1 or 3 for each $X \in\{A, B, C, D\}$ and $D \in \mathcal{D}$. There are essentially only two solutions, as shown in Figs. 3 and 4 . Thus, each fourfold cover of a D-symbol in our list either contains exactly one $\Sigma_{0,1,2}$-subsymbol, which must be isomorphic to the one in Fig. 3, or exactly two $\Sigma_{0,1,2}$-subsymbols, which must both be isomorphic to the one in Fig. 4.

The D-symbol cover corresponding to Fig. 3 is obviously unwound. Incidentally, it is even universal. We read off the permutations $\alpha:=(A, D)(B, C)$ for the pair $(1,2)$, $\beta:=(A, B)(C, D)$ for $(3,2)$ and $\gamma:=(A, C)(B, D)$ for $(5,2)$. From the remaining pairs, we obtain nothing new. Our next task is to determine all possible $\sigma_{3}$-actions which induce permutations that together with $\alpha, \beta$ and $\gamma$ generate $S_{4}$. However, by the regularity condition and the structure of $\mathcal{D}$, the $\sigma_{3}$-action can only lead to one new permutation $\delta$, which must be an involution. Thus $\delta$ must be one of $\alpha, \beta$ and $\gamma$, which together generate 


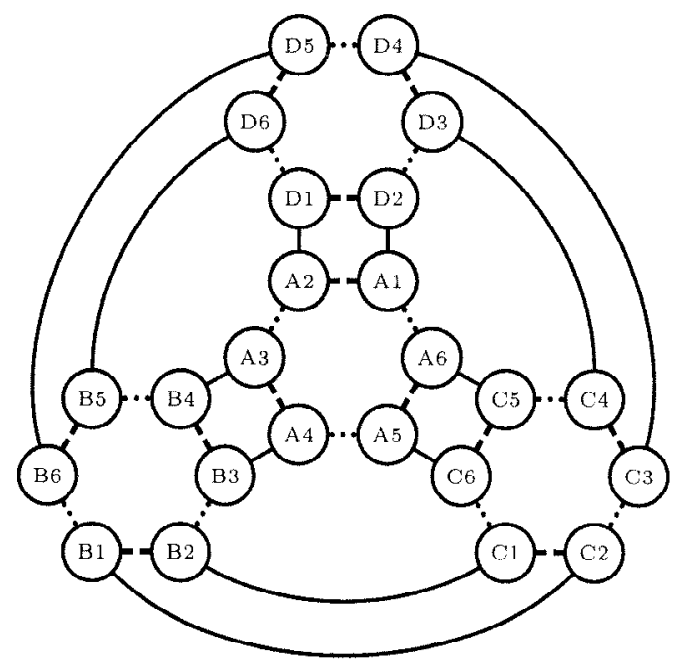

Fig. 3. The universal cover of $\mathcal{D}_{(1,\{0,1,2\})}$.

a four-element group, or a transposition, in which case the generated group has eight elements. Note that this fact is independent of the actual values of $c$ and $d$.

It remains to consider the case where the $\Sigma$-preimage of $\mathcal{D}$ contains two $\Sigma_{0,1,2}$-orbits, each of which is isomorphic to the $\Sigma$-set shown in Fig. 4 . There are three essentially different labellings for this set, according to whether the $\Sigma_{0,2}$-orbits of size 2 are mapped onto $\{1,2\},\{3,4\}$ or $\{5,6\}$, respectively. In any case, we read off no non-trivial permutations from the $\sigma_{0}$ - and $\sigma_{1}$-actions and just one from the $\sigma_{2}$-action, which by appropriate labelling can be assumed to be $(A, B)(C, D)$. Again, we obtain at most one more permutation from the $\sigma_{3}$-action, which must be of order 2. In order to generate $S_{4}$ together with $(A, B)(C, D)$, this must be a transposition other than $(A, B)$ and $(C, D)$. Again by appropriate labelling, we may assume that this transposition is $(B, C)$.

Now by constructing the unique $\Sigma$-set corresponding to each of the nine possible combinations of the second parts of the labels and eliminating isomorphic copies, one

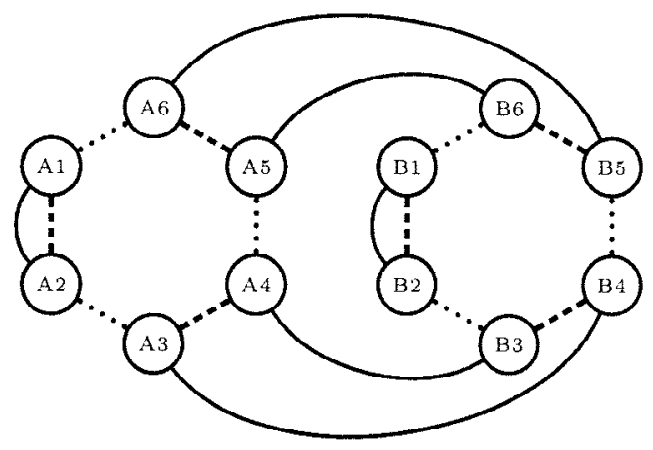

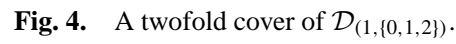




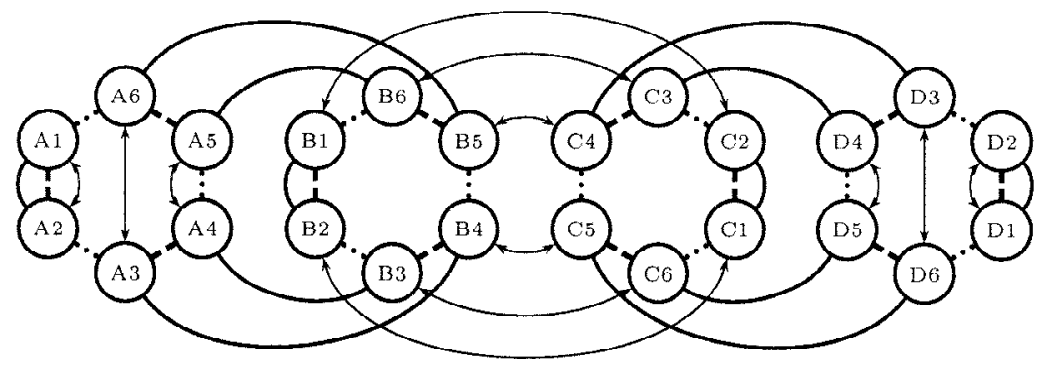

Fig. 5. Case I.

easily checks that there are exactly four $\Sigma$-preimages of $\mathcal{D}$ to be considered, namely those depicted in Figs. 5-8. For each of these and each of our six candidate D-symbols, we can now check whether the $\Sigma$-map onto $\mathcal{D}$ extends to a D-symbol covering, i.e., whether the $r_{2,3}$-values which can be read off from the $\Sigma$-set divide the corresponding $m_{2,3}$-values induced by the map. It turns out thus that Case I is compatible with symbols $\left(\mathcal{D}, M_{4,4}\right)$ and $\left(\mathcal{D}, M_{6,4}\right)$ while Case II is compatible only with symbol $\left(\mathcal{D}, M_{4,4}\right)$, Case III with $\left(\mathcal{D}, M_{3,8}\right)$ and Case IV with $\left(\mathcal{D}, M_{4,6}\right)$.

Given a D-symbol $(\mathcal{D}, M)$, a fourfold cover $\left(\mathcal{D}^{\prime}, M^{\prime}\right)$ and a corresponding homomorphism $\varphi: \pi_{1}(\mathcal{D}, M) \rightarrow S_{4}$, our next task is to construct the cover of $(\mathcal{D}, M)$ which corresponds to the kernel of that homomorphism. The following is a very convenient way to do that: to each pair $(D, i) \in \mathcal{D} \times I$, we have associated a permutation of $\{A, B, C, D\}$. Now we define a new $\Sigma$-set $\hat{\mathcal{D}}=S_{4} \times \mathcal{D}$ with the $\Sigma$-action given by $(\beta, D) \sigma_{i}:=\left(\beta \circ \alpha, D \sigma_{i}\right)$, where $\alpha$ is the permutation associated to $(D, i)$. That is, we construct the cover corresponding to a stabilizer with respect to the multiplication action of $\pi_{1}(\mathcal{D}, M)$ on $S_{4}$ induced by the homomorphism $\varphi$, which of course is exactly the kernel of $\varphi$. The neat thing about this construction is that we do not actually have to construct the $\Sigma$-set $\hat{\mathcal{D}}$ and the induced cover $(\hat{\mathcal{D}}, \hat{M})$ to see whether the latter is unwound. For example, in Case I (Fig. 5) of our list of covers, the trivial permutation is associated to each of the pairs $(1,0),(1,3),(2,0)$ and $(2,3)$, which implies $r_{0,3}(\beta, 1)=1$ and thus by regularity $v_{0,3}(\beta, 1)=2$ for every $\beta \in S_{4}$. Similarly, in Case II (Fig. 6), the trivial permutation is associated to each of the pairs $(3,0)$, $(3,3),(4,0)$ and $(4,3)$. Consequently, $(\hat{\mathcal{D}}, \hat{M})$ is not unwound for any of these two cases.

Incidentally, we had already noted that the symbols $\left(\mathcal{D}, M_{4,4}\right)$ and $\left(\mathcal{D}, M_{6,4}\right)$ cannot correspond to tilings with point group $O$, which gives us a second independent reason to reject Cases I and II.

In Case III (Fig. 7), the cover ( $\hat{\mathcal{D}}, \hat{M})$ turns out to be unwound, but the commutator factor group of its fundamental group is $\mathbb{Z}_{2}$ instead of $\mathbb{Z}^{3}$. Indeed, the corresponding D-symbol $\left(\mathcal{D}, M_{3,8}\right)$ encodes a tiling of the 3 -sphere by 288 tetrahedra.

In Case IV (Fig. 8), the cover is also unwound, and in this single case all further tests are successful. Thus, the D-symbol $\left(\mathcal{D}, M_{6,4}\right)$ encodes a tiling of $\mathbb{E}^{3}$. Testing for other possible point groups where necessary does not reveal any other flat symbols, so we may conclude that there is exactly one flat, regular non-degenerate D-symbol with underlying $\Sigma$-set $\mathcal{D}_{0}$. 


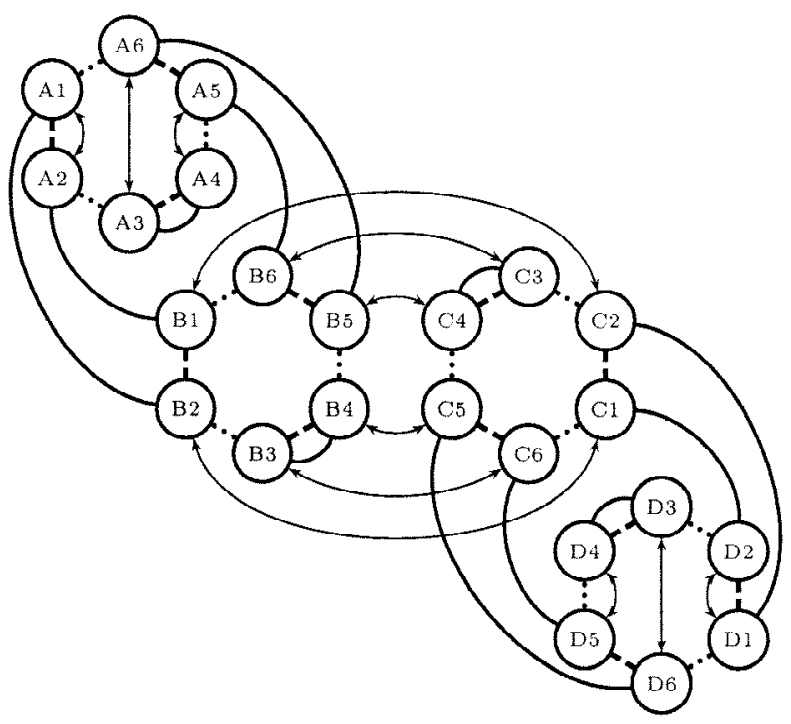

Fig. 6. Case II.

As a final remark, we note that in this particular case, it is easy to check the results geometrically. From the structure of the universal cover of the $\Sigma_{0,1,2}$-subsymbol of $\mathcal{D}_{0}$, shown in Fig. 3, we can deduce that a tiling based on $\mathcal{D}_{0}$ must have combinatorially tetrahedral tiles. Since $\sigma_{3}$ acts trivially, each tile is mapped onto each of its neighbors by a mirror reflection. Thus, all 2 -faces must be flat and the tiles are actually convex tetrahedra with their vertices as extremal points. Furthermore, they possess two opposite edges with dihedral angles of $2 \pi / c$, while all other edges have dihedral angles of $2 \pi / d$. In a euclidean tetrahedron, this is only possible for $c=4$ and $d=6$.

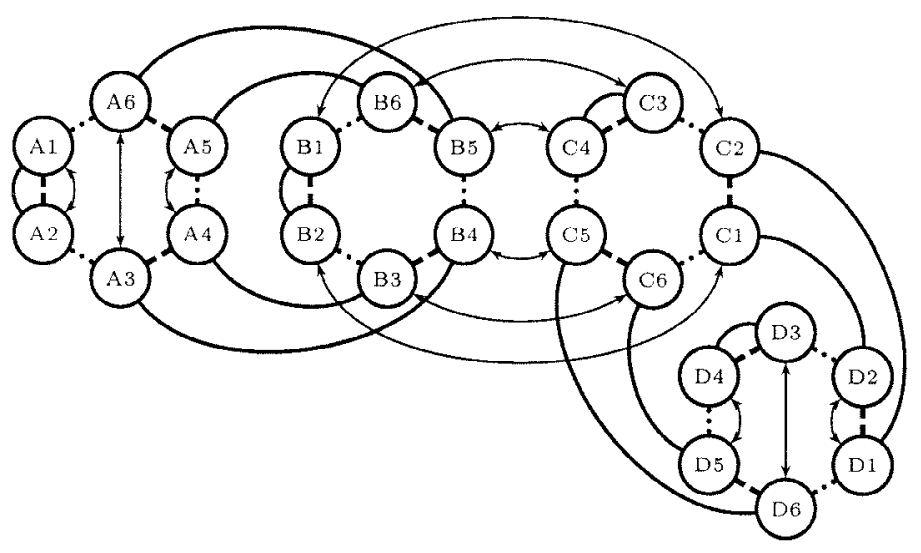

Fig. 7. Case III. 


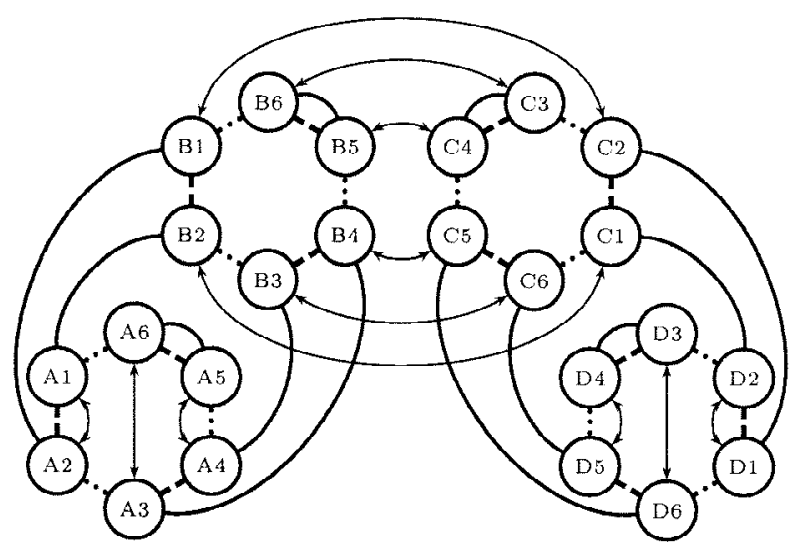

Fig. 8. Case IV.

\section{References}

[BH] L. Balke and D.H. Huson, Two-dimensional groups, orbifolds and tiling, Geom. Dedicata 60 (1996), 89-106.

[Bi] L. Bieberbach, Über die Bewegungsgruppen der Euklidischen Räume II, Math. Ann. 72 (1912), 400.

[BV] L. Balke and A. Valverde, $\Sigma$-Chamber systems, coloured graphs and orbifolds, Contrib. Algebra Geom. 37(1) (1996), 17-29.

$\left[\mathrm{DDH}^{+}\right]$O. Delgado Friedrichs, A.W.M. Dress, D.H. Huson, J. Klinowski, and A.L. Mackay, Systematic enumeration of crystalline networks, Nature 400 (1999), 644-647.

[De1] O. Delgado Friedrichs, Die automatische Konstruktion periodischer Pflasterungen, Master's thesis, University of Bielefeld, 1990.

[De2] O. Delgado Friedrichs, Euclidicity criteria for three-dimensional branched triangulations, Ph.D. thesis, University of Bielefeld, 1994.

[DH1] A.W.M. Dress and D.H. Huson, On tilings of the plane, Geom. Dedicata 24 (1987), 295-310.

[DH2] A.W.M. Dress and D.H. Huson, Heaven and hell tilings, Rev. Topol. Struct. 17 (1991), 25-42.

[DH3] O. Delgado Friedrichs and D.H. Huson, Orbifold triangulations and crystallographic groups, Period. Math. Hungari. 34(1-2) (1997), 29-55.

[DH4] O. Delgado Friedrichs and D.H. Huson, Tiling space by platonic solids I., Discrete Comput. Geom. 21 (1999), 299-315.

[DH5] O. Delgado Friedrichs and D.H. Huson, Four-regular vertex-transitive tilings of $\mathbf{E}^{3}$, Discrete Comput. Geom. 24(2) (2000), 279-292.

[DHM] A.W.M. Dress, D.H. Huson, and E. Molnár, The classification of face-transitive 3d-tilings, Acta Cryst. Sect. A 49 (1993), 806-817.

[DHZ] O. Delgado Friedrichs, D.H. Huson, and E. Zamorzaeva, The classification of 2-isohedral tilings of the plane, Geom. Dedicata 42 (1992), 43-117.

[Dr1] A.W.M. Dress, Regular polytopes and equivariant tessellations from a combinatorial point of view, in Algebraic Topology, Göttingen 1984, pp. 56-72, Lecture Notes in Mathematics, vol. 1172, SpringerVerlag, Berlin, 1985.

[Dr2] A.W.M. Dress, Presentations of discrete groups, acting on simply connected manifolds, Adv. in Math. 63 (1987), 196-212.

[FG] M. Ferri and C. Gagliardi, Crystallization moves, Pacific J. Math. 100 (1982), 85-103.

[Fr] R. Franz, Zum rekursiven Aufbau der Familie der periodischen Pflasterungen der euklidischen Ebene, Ph.D. thesis, University of Bielefeld, 1988.

[Ha1] W. Haken, Verfahren zur Aufspaltung einer 3-Mannigfaltigkeit in irreduzible 3-Mannigfaltigkeiten, Math. Z. 76 (1961), 427-467. 
[Ha2] W. Haken, Über das Homöomorphieproblem der 3-Mannigfaltigkeiten, Math. Z. 80 (1962), 89-120.

[He1] J. Hempel, 3-Manifolds, Princeton University Press, Princeton, NJ, and University of Tokyo Press, Tokyo, 1976.

[He2] G. Hemion, The Classification of Knots and 3-Dimensional Spaces, Oxford University Press, Oxford, 1992.

[Hu1] D.H. Huson, Patches, stripes and net-like tilings, Ph.D. thesis, University of Bielefeld, 1989.

[Hu2] D.H. Huson, The generation and classification of tile- $k$-transitive tilings of the euclidean plane, the sphere and the hyperbolic plane, Geom. Dedicata 47 (1993), 269-296.

[Hu3] D.H. Huson, Tile-transitive partial tilings of the plane, Contrib. Geom. Algebra 34(1) (1993), 87-118.

[Li] S. Lins, Gems, Computers and Attractors for 3-Manifolds, Series on Knots and Everything, vol. 5, World Scientific, Singapore, 1995.

[LM] S. Lins and A. Mandel, Graph-encoded 3-manifolds, Discrete Math. 57(3) (1985), 261-284.

[Ma] S. V. Matveev, Computer recognition of three-manifolds, Exper. Math. 7(2) (1998), 153-161.

[MS] W. Meeks and P. Scott, Finite group actions on 3-manifolds, Invent. Math. 86 (1986), 287-346.

[Ne] J. Neubüser, An elementary introduction to coset table methods in computational group theory, Proceedings of the Groups St. Andrews Conference, 1981, pp. 1-45.

[NF] J. Neubüser and V. Felsch, Personal communication, 1993.

[Ni] W. Nickel, Central extensions of polycyclic groups, Ph.D. thesis, Australian National University, Canberra, 1993.

[Ru] J.H. Rubinstein, An algorithm to recognize the 3-sphere, Proceedings of the International Congress of Mathematicians (Zürich, 1994), vols. 1, 2 (1995), pp. 601-611.

[Sc] P. Schmitt, $n$-homeohedral types of tilings, Geom. Dedicata 32(3) (1989), 319-327.

[Th1] Abigail Thompson, Thin position and the recognition problem for S3, Math. Res. Lett. 1(5) (1994), 613-630.

[Th2] W.P. Thurston, The Geometry and Topology of Three-Manifolds, Princeton University, Princeton, NJ, 1980, Chapter 13.

[Th3] W.P. Thurston, Three-Dimensional Geometry and Topology, Vol. 1, Princeton University Press, Princeton, NJ, 1997 (Edited by Silvio Levy).

[Vi] E.B. Vinberg (ed.), Geometry II: Spaces of Constant Curvature, Encyclopaedia of Mathematical Sciences, vol. 29, Springer-Verlag, Berlin, 1993.

Received June 20, 2000, and in revised form October 25, 2000. Online publication May 4, 2001. 